\title{
Genome-wide screening and functional analysis identify a large number of long noncoding RNAs involved in the sexual reproduction of rice
}

Yu-Chan Zhang ${ }^{1 \dagger}$, Jian-You Liao ${ }^{2 \dagger}$, Ze-Yuan Li ${ }^{1}$, Yang Yu', Jin-Ping Zhang ${ }^{1}$, Quan-Feng Li', Liang-Hu Qu', Wen-Sheng Shu ${ }^{1}$ and Yue-Qin Chen ${ }^{1 *}$

\begin{abstract}
Background: Long noncoding RNAs (IncRNAs) play important roles in a wide range of biological processes in mammals and plants. However, the systematic examination of IncRNAs in plants lags behind that in mammals. Recently, IncRNAs have been identified in Arabidopsis and wheat; however, no systematic screening of potential IncRNAs has been reported for the rice genome.

Results: In this study, we perform whole transcriptome strand-specific RNA sequencing (ssRNA-seq) of samples from rice anthers, pistils, and seeds 5 days after pollination and from shoots 14 days after germination. Using these data, together with 40 available rice RNA-seq datasets, we systematically analyze rice IncRNAs and definitively identify IncRNAs that are involved in the reproductive process. The results show that rice IncRNAs have some different characteristics compared to those of Arabidopsis and mammals and are expressed in a highly tissue-specific or stage-specific manner. We further verify the functions of a set of IncRNAs that are preferentially expressed in reproductive stages and identify several IncRNAs as competing endogenous RNAs (ceRNAs), which sequester miR160 or miR164 in a type of target mimicry. More importantly, one IncRNA, XLOC_057324, is demonstrated to play a role in panicle development and fertility. We also develop a source of rice IncRNA-associated insertional mutants.

Conclusions: Genome-wide screening and functional analysis enabled the identification of a set of IncRNAs that are involved in the sexual reproduction of rice. The results also provide a source of IncRNAs and associated insertional mutants in rice.
\end{abstract}

\section{Background}

Non-protein-coding RNAs (ncRNAs) constitute a substantial portion of transcribed sequences with structural, regulatory or unknown functions. Because of these important biological roles, ncRNAs have been of great research interest in recent years. Attention was previously given to small regulatory RNAs (sRNAs), such as microRNAs (miRNAs), which are less than 200 nucleotides in length [1]. ncRNAs longer than 200 nucleotides (long non-coding RNAs (lncRNAs)) were found to have functions associated with virtually every biological process in

\footnotetext{
* Correspondence: Isscyq@mail.sysu.edu.cn

${ }^{\dagger}$ Equal contributors

'Key Laboratory of Gene Engineering of the Ministry of Education, State Key Laboratory for Biocontrol, School of Life Science, Sun Yat-Sen University, Guangzhou 510275, PR China

Full list of author information is available at the end of the article
}

mammals, and these initial reports initiated a wave of research on lncRNAs that followed the path of sRNA research. Recently, lncRNAs have emerged as potent regulators, particularly in mammals. However, studies on IncRNAs in plants remain at the early stage; only a few lncRNAs have been shown to regulate plant development, especially during reproduction [2].

Sexual reproduction is one of the most essential biological processes and occurs in a vast number of species. Numerous studies have been devoted to the identification of reproduction-related genes, making great progress in understanding the reproductive processes of both animals and plants. However, the complex regulatory networks involving these genes remain largely unknown. Intriguingly, many lncRNAs have recently been proven to play important roles in reproductive processes through the regulation of related genes in various species. In mammals, lncRNAs, 
such as Xist, H19, Kcnq1ot1, bxd and HOTAIR, have been found to be crucial for the precise control of embryogenesis [3-6]. Notably, several plant lncRNAs have also been demonstrated to participate in reproductive regulation, including COLDAIR, COOLAIR, LDMAR, CsM10 and Zm401 [7-11], indicating that one of the principal functions of plant lncRNAs might be to regulate plant reproduction. More interestingly, Komiya et al. [12] found that a number of large intergenic non-coding RNAs (lincRNAs) could generate 21-nucleotide phasiRNAs, which associate with the germline-specific Argonaute (AGO) proteinMEL1 in rice, indicating that rice lncRNAs might play a role in the development of pre-meiotic germ cells. Genome-wide analysis is necessary to discover new lncRNAs and is important for the further functional analysis of these RNAs. More than 8,000 lncRNAs have been identified in humans using bioinformatic methods [13], and approximately 4,000 lncRNAs have been identified in mice [14,15]. In plants, 6,480 transcripts have been classified as IncRNAs in Arabidopsis [16,17], and 125 putative stress-responsive lncRNAs have been identified in wheat [18]. Although rice is a model species for plant development studies and represents a staple food for nearly half of the global population, rice lncRNAs remain poorly characterized, and no systematic screening of potential lncRNAs in the rice genome has been reported.

In this study, we performed whole transcriptome strandspecific RNA sequencing (ssRNA-seq) of samples obtained from rice anthers, pistils, seeds that were harvested 5 days after pollination (DAP) and shoots that were harvested 14 days after germination (DAG). Together with 40 available rice RNA-seq datasets, we systematically identified rice lncRNAs (including lincRNAs and antisense lncRNAs) with a specific focus on the lncRNAs that were expressed at reproductive stages and performed functional studies on some of these reproduction related lncRNAs. Our results indicated that a number of rice lncRNAs are highly tissuespecific, and a large portion of these RNAs are specifically expressed in conjunction with reproduction-related processes, particularly in pollen.

\section{Results}

A computational approach for the genome-wide identification of IncRNAs in rice

To systematically identify lncRNAs related to rice reproduction, we performed whole transcriptome ssRNAseq of rice anthers, pistils, seeds that were harvested 5 DAP and shoots that were harvested 14 DAG (the sequencing results included $3.89 \times 10^{8}$ reads; Additional file 1; Sequence Read Archive (SRA) accession number SRP047482). We then developed a rice IncRNA computational identification pipeline based on RNA-seq data (Figure 1) using 4 whole transcriptome ssRNA-seq data sets and 40 available poly (A) RNA-seq data sets $\left(1.23 \times 10^{9}\right.$ reads). These datasets covered most of the organs and stages involved in rice reproduction (Additional file 1) and were suitable for the identification of reproduction-related lncRNAs. Our IncRNA identification strategy comprised three key procedures (Figure 1). First, the rice transcriptome was reconstructed from all of the RNA-seq datasets using Cufflink 2.0 [19]. After filtering out infrequently expressed transcripts (those showing FPKM (fragments per kilobase of transcript per million mapped reads) scores $<0.5$ in all samples) and transcripts without strand information, we recovered $77.4 \%(30,219 / 39,045)$ of the non-transposableelement (non-TE)-related mRNAs in the datasets (the mRNAs discussed in the following sections are non-TE-related mRNAs unless otherwise specified). The efficient recovery of known protein-coding genes indicated that the dataset employed here was suitable for the recovery of novel transcribed regions of the rice genome.

Second, we only retained novel (not overlapping with known genes in sense), large (longer than 200 nucleotides), expressed (for multiple-exon transcripts FPKM $\geq 0.5$, for single-exon transcripts FPKM $\geq 2$ ) transcripts. All singleexon transcripts close to other transcripts were removed. We then evaluated the coding potential of the remaining transcripts and obtained novel expressed lncRNAs. We used the Coding Potential Calculator (CPC) [20] to predict the coding potential of each transcript. All transcripts with $\mathrm{CPC}$ scores $>0$ were discarded. To guarantee the thorough elimination of protein-coding transcripts, we also employed HMMER [21] to scan each transcript with a CPC score $<0$ in all three reading frames to exclude transcripts that encoded any of the known protein domains cataloged in the Pfam protein family database [22]. Finally, we obtained 2,224 reliably expressed lncRNAs (Additional file 2), including 1,624 lincRNAs and 600 long non-coding natural antisense transcripts (lncNATs), which intersect any exon of a protein-coding mRNA on the opposite strand.

\section{The genomic characteristics and conservation of rice IncRNAs}

We characterized the basic genomic features of the obtained lncRNAs and compared these features with the available features of Arabidopsis or human lncRNAs or to rice protein-coding genes where appropriate. We found that only a small fraction (median percentage, $6.5 \%$ ) of the sequence of most of the lncNATs was antisense overlapped by protein-coding mRNA (Figure 2A) and that lincRNAs and lncNATs are similar in many aspects (Figure 2). To display the characteristics of lincRNAs and lncNATs more clearly, we analyzed the characteristics of lincRNAs and lncNATs separately in the following comparisons. Similar to findings for Arabidopsis [16,23], only around half of lncRNAs were spliced (46.5\% for lincRNAs, $65.9 \%$ for $\operatorname{lncNATs}$ ). In contrast, more than $98 \%$ of human lncRNAs are spliced [24] (Figure 2B). Rice lncRNAs have 


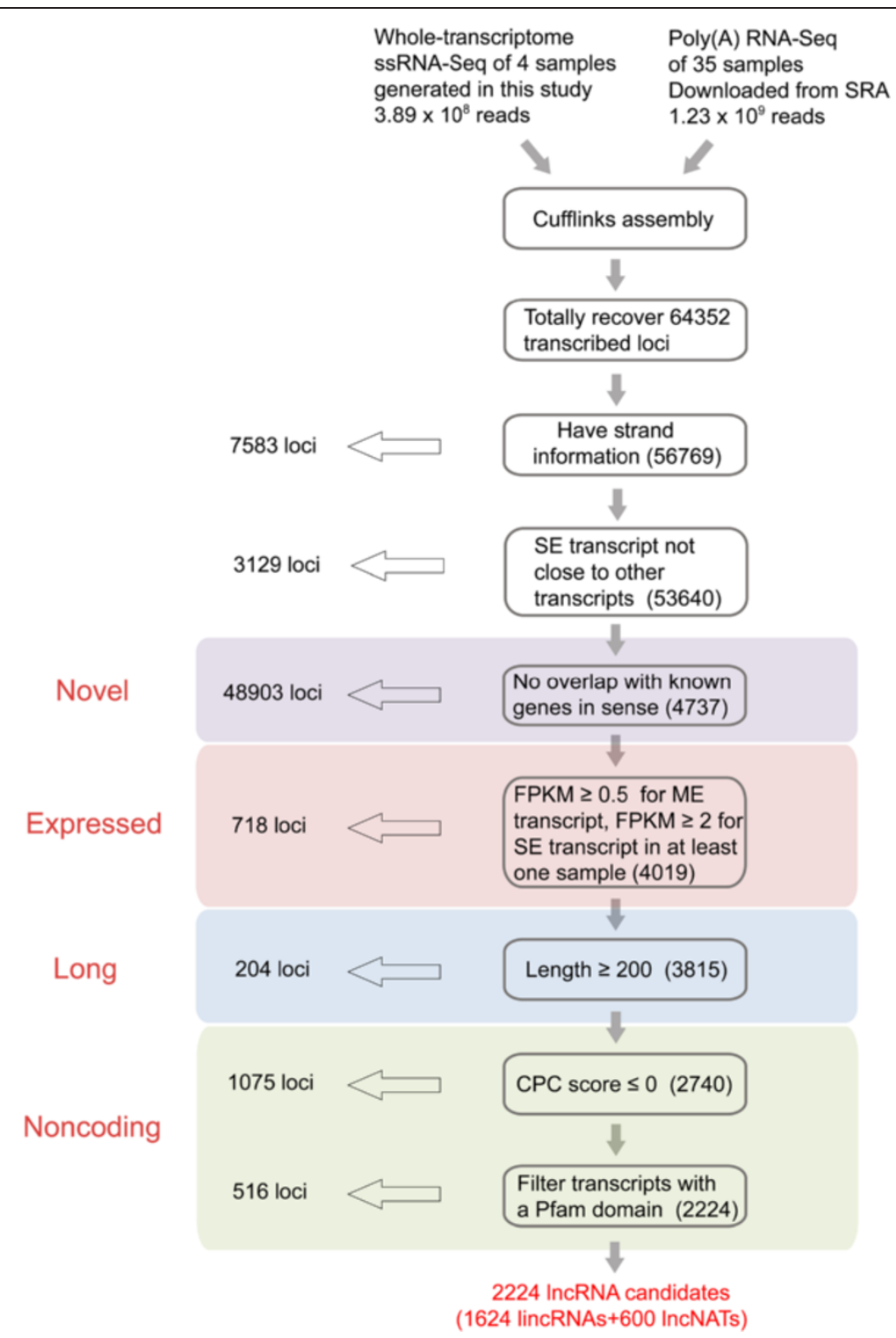

Figure 1 An integrative computational pipeline for the systematic identification of IncRNAs in rice. CPC,Coding Potential Calculator; IncNAT, long non-coding natural antisense transcript; ME, multiple exon; SE, single exon.

fewer exons than mRNAs (2.21 versus 4.67 on average, respectively; 2.10 exons for lincRNAs and 2.42 exons for lncNATs), but their exon lengths (median length of 323 nucleotides; 322 nucleotides for lincRNAs, 298 nucleotides for lncNATs) are longer than those of mRNA (median length of 159 nucleotides) (Figure 2C). Full-length rice lncRNA transcripts (median length of852 nucleotides; 800 nucleotides for lincRNAs, 950 nucleotides for lncNATs) are longer than Arabidoposis lncRNA transcripts (median length of 285 nucleotides) [16,23] and human lncRNA transcripts (median length of 592 nucleotides) [24], and are generally shorter than protein-coding transcripts (median length of 1,411 nucleotides) (Figure 2D). Rice lncRNAs generally do not overlap with repeat sequences (Figure 2E); fewer repeats-overlapped rice lncRNAs than repeatsoverlapped rice mRNAs and repeats-overlapped human lncRNAs. Like Arabidopsis lncRNAs [16,23], only a small proportion of rice lncRNAs (122 of 1,624 lincRNAs, 7.5\%; 44 of 600 lncNATs, 7.3\%) generate sRNAs (Additional file 3), implying that these lncRNAs might function through generating sRNAs. Interestingly, rice lncRNAs were much more A/U-rich than the coding sequences and the 5'UTRs of protein-coding genes but were less A/U-rich than 3' UTRs that use A/U-rich elements to regulate mRNA degradation [25] (Figure 2F). This characteristic is conserved in Arabidopsis (Figure S1A in Additional file 4) and animal lncRNAs [26,27], implying that this feature might be related to the functions of lncRNAs. Rice lincRNAs are most likely 


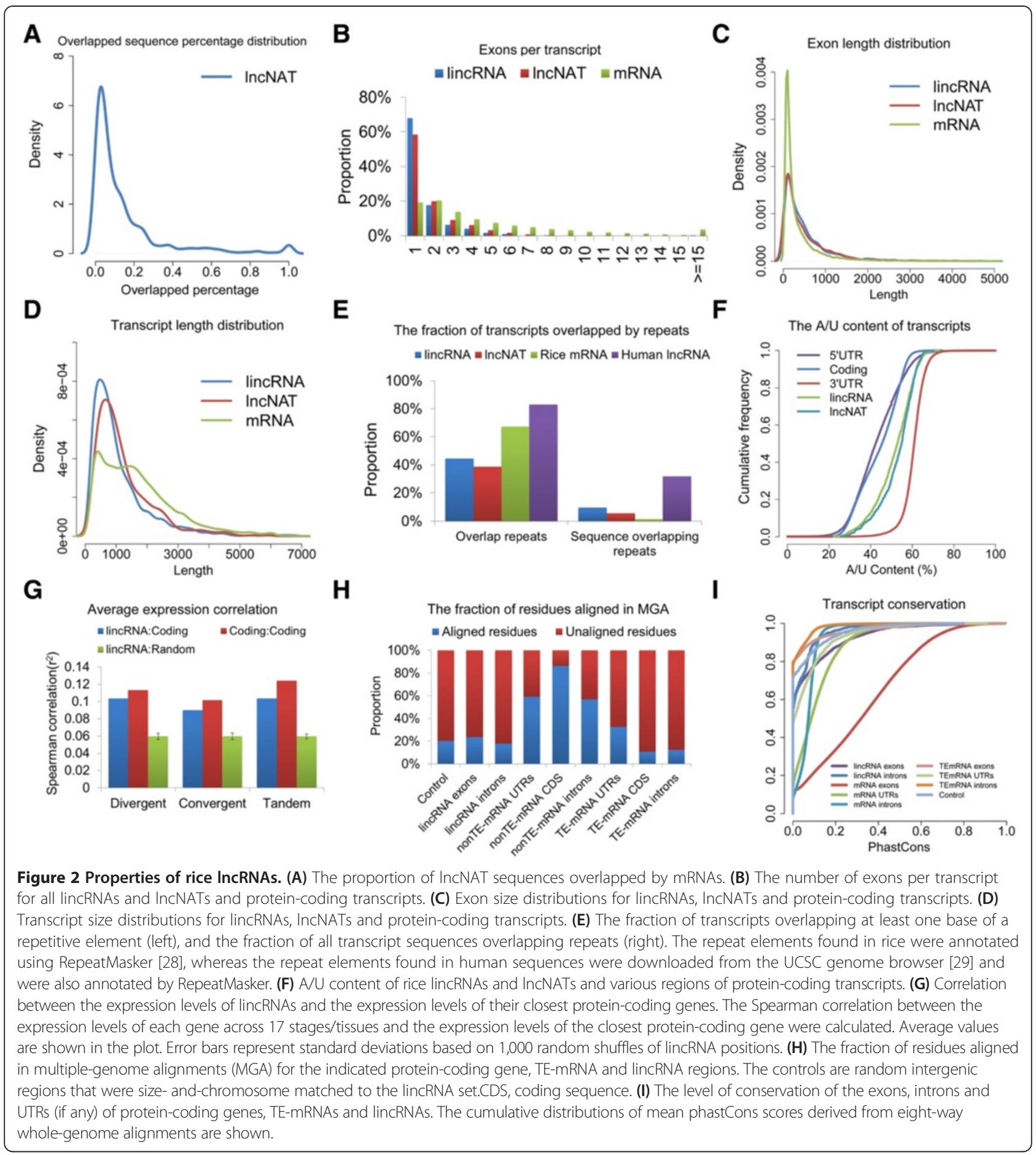

to appear in divergent orientations with respect to the closest neighboring protein-coding genes (Figure S1B in Additional file 4). However, we did not observe a stronger correlation between the expression of rice lincRNAs and their nearest neighbors than that between adjacent protein-coding genes (Figure 2G), although the expression of lncNATs is more highly correlated with convergent and divergent overlapped mRNA than with tandem overlapped mRNAs (Figure S1C in Additional file 4).

We further analyzed the conservation of rice lncRNAs through an eight-way genomic alignment between the genomes of rice [30], Musa [31], Arabidopsis [32], Brachypodium [33], maize [34], poplar [35], grapevine [36] and Sorghum [37] using MultiZ [38]. Our analysis mainly 
focused on lincRNAs because the partial antisense overlap of lncNATs might have interfered with the analysis. The genomes were then aligned to determine the conservation score (consScore) of each nucleotide in the rice genome using phastCons [39]. The fraction of lincRNA residues that aligned in the whole-genome alignments was $23.5 \%$; this value is much higher than those of TE-mRNA coding sequences (10.7\%) and TE-mRNA introns (12.3\%) but is much smaller than those of mRNA coding sequences (86.3\%), UTRs (59.1\%) and introns (56.9\%) and is comparable to those of lincRNA introns (18.0\%) and intergenic controls (20.2\%) (Figure 2H). We further measured the conservation of lincRNAs based on the obtained consScores. The exons of lincRNAs were more conserved than the introns of lincRNAs and control exons with matched lengths (Figure 2I). Interestingly, both the exons and introns of the studied lincRNAs were more conserved than those of TE-related mRNAs. However, the rice lincRNAs were less conserved than mRNA introns, possibly due to the presence of conserved ncRNAs (such as small nucleolar RNAs (snoRNAs)) in mRNA introns [40]. Previous studies have shown that both plant and animal lncRNAs can function through short conserved regions (despite the rapid sequence evolution observed elsewhere in these RNAs) and have indicated that lincRNAs in animals are likely to contain short conserved regions [26,27,41,42]. However, we found that rice lincRNAs do not contain shorter conserved regions than protein-coding genes (Figure S1D in Additional file 4), suggesting that lincRNAs that function through short conserved regions might not be as common in rice as in animals.

Rice IncRNAs are highly tissue-specific, and many IncRNAs are specifically expressed during reproduction

We then estimated the expression level of each transcript using FPKM and found that the lincRNAs and lncNATs were expressed at similar levels (median: 8.0 FPKM versus 7.21 FPKM, respectively), which were lower than the levels at which protein-coding genes are expressed (median: 19.3 FPKM, both $P<2.2 \times 10^{-16}, t$-test) but higher than the levels at which TE-related mRNAs are expressed (median: 4.2 FPKM, both $P<2.2 \times 10^{-16}, t$-test) (Figure $3 \mathrm{~A}$ ). We

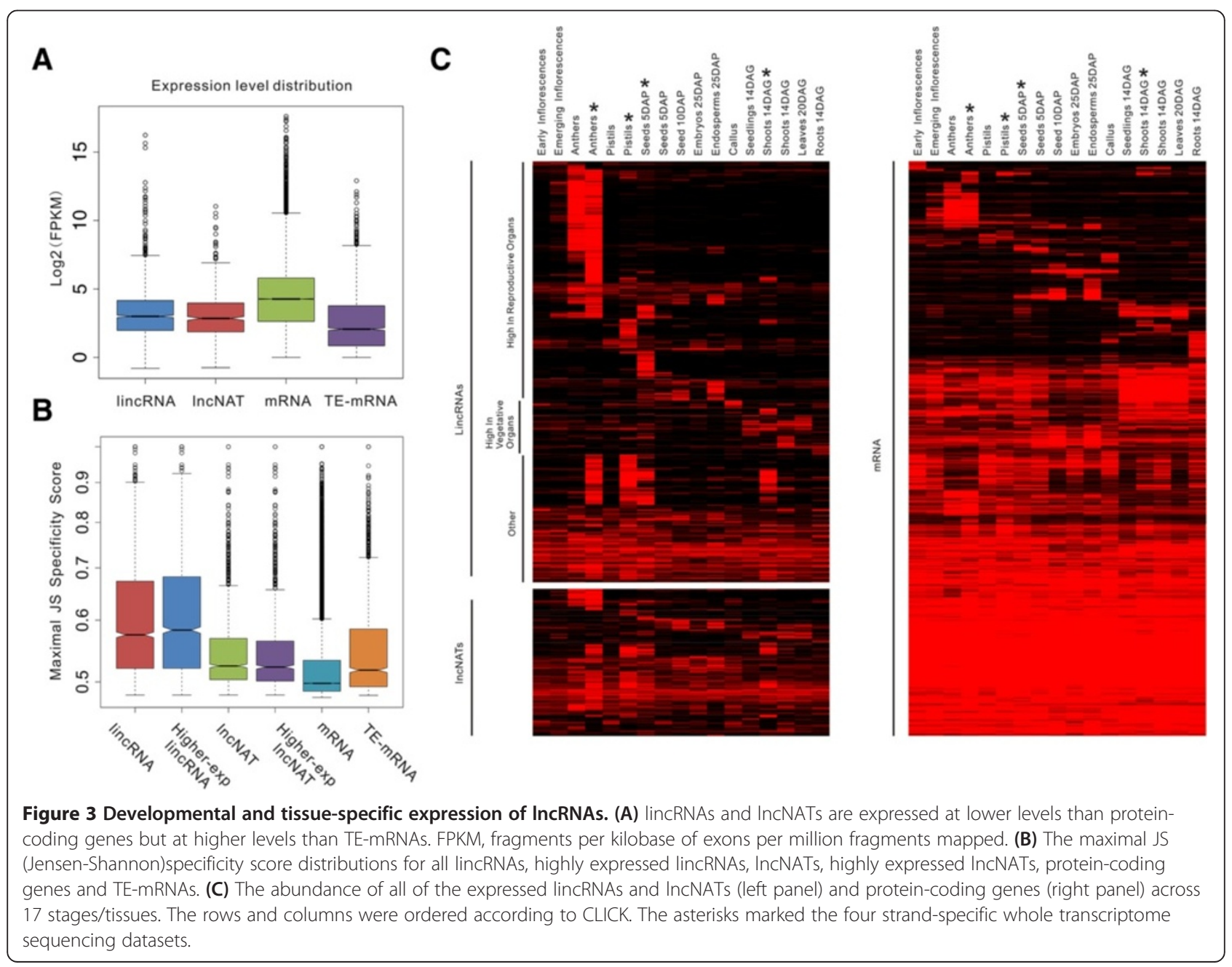


further estimated the degree of the differential expression of lincRNAs, lncNATs, mRNAs and TE-related mRNAs based on the JS (Jensen-Shannon) score [13]. Intriguingly, we found that lincRNAs tend to be far more differentially expressed than IncNATs $\left(P<2.2 \times 10^{-16}\right.$, KolmogorovSmirnovtest), which exhibited a similar degree of differentiated expression to that of TE-mRNAs; both lincRNAs and lncNATs are more differentially expressed than mRNAs (both $P<2.2 \times 10^{-16}$, Kolmogorov-Smirnovtest) (Figure 3B). The lower expression level and highly differentiated expression pattern of lincRNAs were also found in Arabidopsis and animals $[13,16]$, suggesting that both of these characteristics are conserved for lincRNAs.

The highly tissue-specific expression pattern observed for lincRNAs suggests that it might be possible to classify lincRNAs according to their expression patterns. We clustered the lincRNAs based on their expression patterns in 13 different types of tissue samples using CLICK [43]. Remarkably, the lncRNAs can be classified into three categories: lincRNAs that are highly expressed in reproductive organs (including panicles, anthers, pistils, seeds 5DAP, seeds 10DAP, embryos 25DAP and endosperms 25DAP); lincRNAs that are highly expressed in vegetative organs (including callus, seedlings 14DAG, shoots 14DAG, leaves 20DAG and roots 14DAG), and other lincRNAs (lincRNAs expressed in multiple organs or only expressed in our sequencing datasets); lncNATs were separately categorized (Figure 3C; Additional file 2).

Interestingly, we found that a number of rice lncRNAs are specifically expressed at a single development stage, and this type of lncRNA was expressed during the integrated sexual reproduction process (Figure 3C; Additional file 2), indicating that lncRNAs may function throughout the entire reproductive process in rice. To confirm the expression patterns of the lncRNAs, we randomly selected 10 lncRNAs, including both single-exon lncRNAs (XLOC_045319, XLOC_016182) and multi-exonic lncRNAs (XLOC_018316, XLOC_037529, XLOC_057981, XLOC_040350, XLOC_010670, XLOC_009232, XLOC_ 053418 and XLOC_004275) (Figures 4 and 5), and validated their expression patterns using real-time quantitative PCR (qRT-PCR). We found a nearly perfect concordance between our experimental results and the RNA-seq results for most of the studied tissues, suggesting that the lncRNA expression patterns based on RNA-seq data are reliable.

We also performed in situ hybridization to analyze the spatial expression patterns of 4 of these 10 validated lncRNAs that were preferentially expressed during reproductive stages and that exhibited high abundance and conservation (Figure 5). Interestingly, these lncRNAs exhibited higher degrees of tissue specificity in their expression patterns than we observed in their expression profiles, and their expression tended to be restricted to particular types of cells - for example, XLOC_010670 and XLOC_004275 are highly expressed in sperm (Figure 5A, D); XLOC_053418 is specifically expressed in ovules (Figure 5B); and XLOC_009232 is specifically expressed in coleoptiles (Figure 5C) - rather than being ubiquitously expressed throughout the tissue.

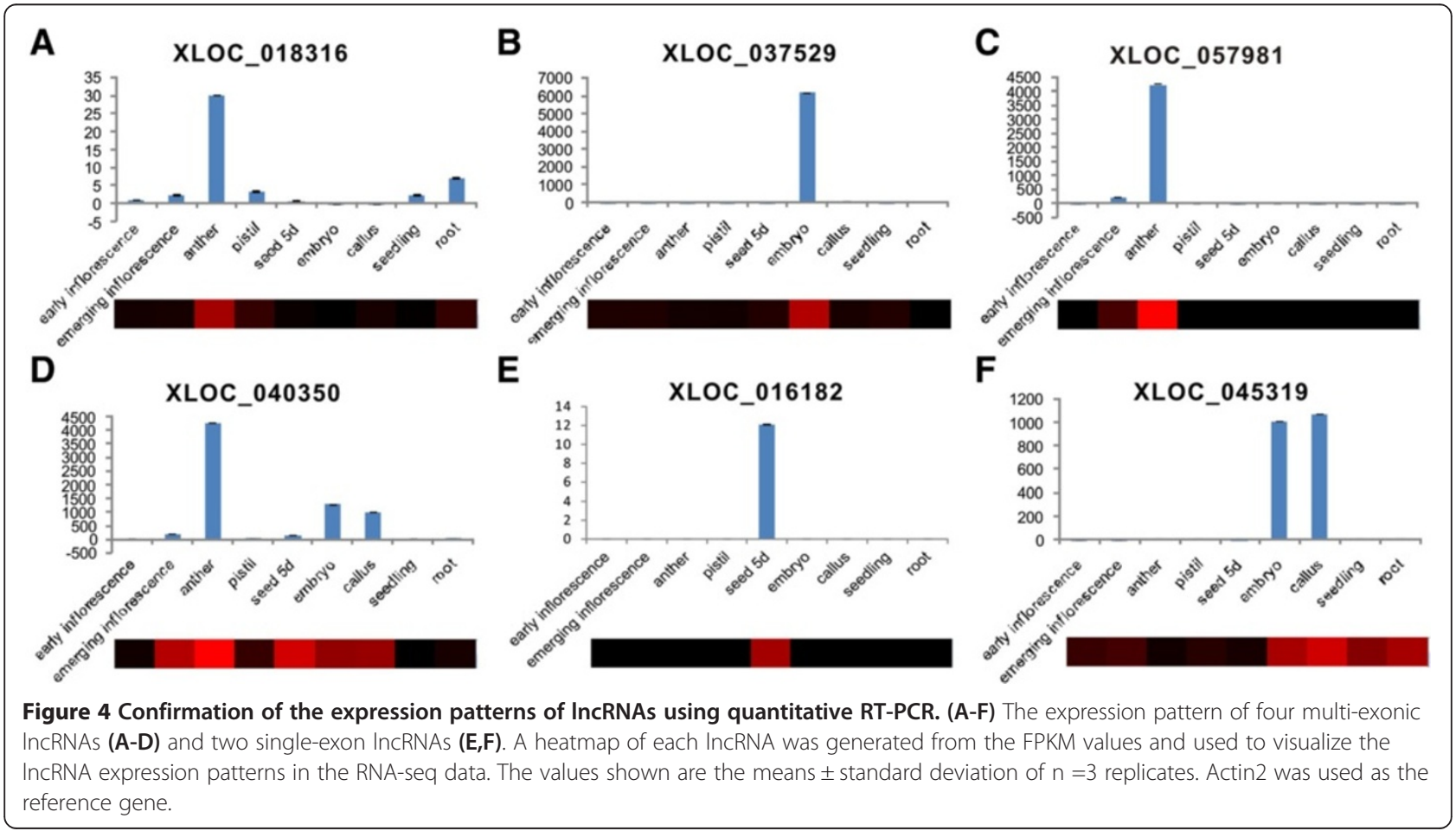




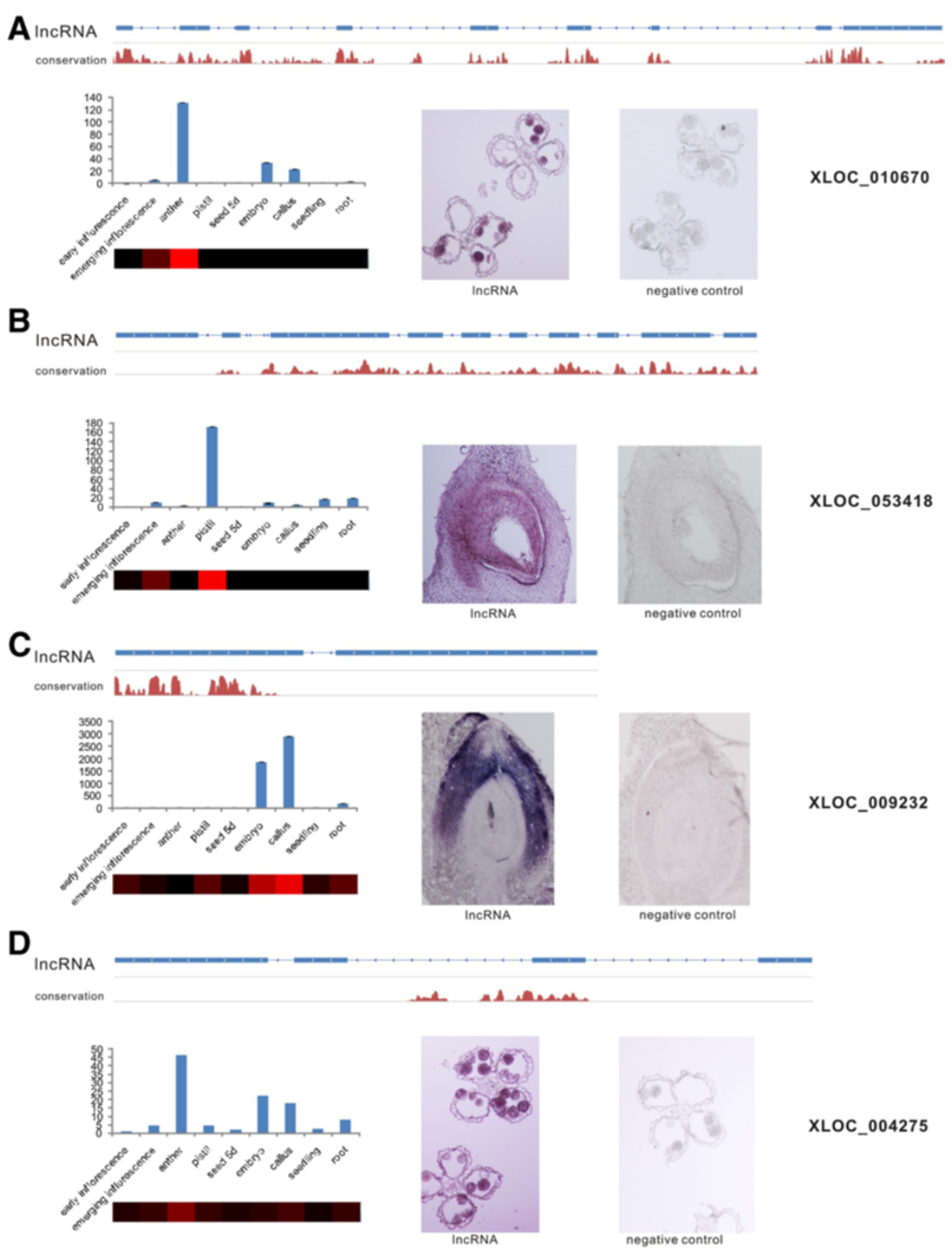

Figure 5 Expression pattern and spatial expression pattern analysis of four conserved IncRNAs based on qRT-PCR and in situ hybridization. (A-D) The top part of each panel shows the conservation of a particular IncRNA; the lower left of each panel shows the expression pattern of the IncRNA based on qRT-PCR, and the lower right of each panel shows the spatial expression pattern in sperm $(\mathbf{A}, \mathbf{D})$ coleoptile (B) or ovule (C) of the particular IncRNA examined by in situ hybridization. The antisense transcript of each IncRNA was used to detect the expression of corresponding IncRNAs by in situ hybridization, and the antisense transcripts of IncRNAs that were not expressed in the corresponding organs were used as a negative control. A heatmap of each IncRNA was generated from the FPKM values and was used to visualize the IncRNA expression patterns in the RNA-seq data. The values shown are the means \pm standard deviation ( $n=3$ replicates). Actin2 was used as the reference gene.

Of the identified reproduction-related lincRNAs, most were specifically expressed in anther (58.0\%) (Figure 3C). This finding is similar to previous observations obtained in animals: a large proportion of lincRNAs are specifically expressed in the testis [13]. However, this phenomenon was not apparent for rice lncNATs. Thus, this characteristic might be specific for lincRNAs, which suggests that lincRNAs play important and conserved roles in male gametophyte genesis and in the regulation of reproductive growth. To further investigate whether the lincRNAs that are specifically expressed during reproduction have corresponding functions, we performed gene ontology (GO) analyses of mRNAs for which the expression patterns were correlated with lincRNAs that were highly expressed in reproductive organs and those that were correlated with lincRNAs that were highly expressed in vegetative organs. 
We found that the mRNA group exhibiting expression patterns correlated with 'reproductive lincRNAs' were significantly enriched in reproduction-specific GO terms, whereas the mRNA group correlated with 'vegetative lincRNAs' did not show such enrichment (Figure 3D; Additional file 5), indicating that lincRNAs that are specifically expressed during the reproductive process might function in regulating reproductive growth.

\section{Insertional mutant analysis reveals a set of lincRNAs that participate in reproduction}

The results described above suggest that a set of lncRNAs might be associated with the regulation of reproduction. To investigate the functions of rice lncRNAs in the regulation of reproduction, we first performed a preliminary functional analysis of all of the identified lincRNAs in association with all nine existing rice mutant databases, including the affjp [44,45], cirad [46,47], gsnu, ostid [48], pfg [49], rmd [50], ship, trim and ucd databases (Additional file 6). IncNATs were not selected for mutant analysis because they are partially overlapped with protein-coding genes, which might produce false positive results. Our strategy was to blast the flanking sequence tags (FSTs) included in each mutant database against the 1,624 lincRNAs and their 1-kb upstream regions separately. A total of 736 lincRNA-related insertional mutants were found in these databases (Additional file 7). Among these mutants, 233 lincRNAs were related to mutants with insertions in their transcribed regions, and 227 were related to mutants with insertions in their potential promoter regions, as determined from at least one mutant database (Table 1). These mutants would contribute to the prospective functional analysis of individual lincRNAs.

Of the nine available rice mutant databases, only affjp includes published phenotypic data [45], and this database was therefore selected for use in a further analysis of the relationships between the expression patterns of the
lincRNAs and their phenotypes. Among the 84 mutants found in the affjp database, 47 exhibited Tos17 insertions in the transcribed regions of 30 lincRNAs, whereas 37 showed insertions in the potential promoter regions of 19 lincRNAs. Moreover, $76.7 \%$ of the lincRNAs with insertions in their transcribed regions and $73.7 \%$ of the lincRNAs with insertions in their promoter regions are associated with observed phenotypes. The phenotypes related to these lincRNAs have been summarized and are presented with their expression patterns to allow readers to search for lincRNAs of interest in Additional file 8 .

We divided the observed phenotypes into two groups: phenotypes related to reproductive growth, such as low fertility, sterility, abnormal panicles and heading dates; and phenotypes related to vegetative growth, including height, tillering, lethality, germination and leaf-related phenotypes. Of these mutants, $45.9 \%$ possess phenotypes related to reproductive growth for the transcribed region insertional mutants, and $45.0 \%$ possess phenotypes related to reproductive growth for the promoter region insertional mutants (Additional file 8). Intriguingly, more than $80 \%$ of the lincRNAs that were highly expressed in reproductive organs caused phenotypes related to reproductive growth with insertions in either transcribed regions or promoter regions, and only 30 to $40 \%$ of the lincRNAs that belong to the 'other' group (with unbiased expression patterns) caused phenotypes related to reproductive growth (Figure 6A,B). This finding provides further support for the notion that lincRNAs that are preferentially expressed at the reproductive stage regulate reproductive growth.

\section{Functional analysis of several reproduction-related IncRNAs elucidated their roles as competing endogenous RNAs or participants in the regulation of reproduction} It has been shown that lncRNAs function as competing endogenous RNAs (ceRNAs) by binding to and sequestering

Table 1 Numbers of available lincRNA-correlated rice mutants

\begin{tabular}{|c|c|c|c|c|c|c|}
\hline \multirow[t]{2}{*}{ Mutant database $^{a}$} & \multicolumn{3}{|l|}{ Internal } & \multicolumn{3}{|c|}{ Upstream } \\
\hline & $\begin{array}{l}\text { Mutant } \\
\text { number }\end{array}$ & $\begin{array}{l}\text { IncRNA } \\
\text { number }\end{array}$ & $\begin{array}{l}\text { With phenotype } \\
\text { information }\end{array}$ & $\begin{array}{l}\text { Mutant } \\
\text { number }\end{array}$ & $\begin{array}{l}\text { IncRNA } \\
\text { number }\end{array}$ & $\begin{array}{l}\text { With phenotype } \\
\text { information }\end{array}$ \\
\hline affjp & 47 & 30 & 27 & 37 & 19 & 14 \\
\hline cirad & 178 & 117 & 73 & 209 & 131 & 90 \\
\hline gsnu & 3 & 3 & - & 6 & 4 & - \\
\hline ostid & 2 & 2 & - & 1 & 1 & - \\
\hline $\mathrm{pfg}$ & 29 & 30 & - & 12 & 12 & - \\
\hline rmd & 16 & 13 & - & 8 & 7 & - \\
\hline ship & 3 & 3 & - & 2 & 1 & - \\
\hline trim & 76 & 62 & - & 72 & 59 & - \\
\hline ucd & 13 & 11 & - & 22 & 12 & - \\
\hline
\end{tabular}

${ }^{\mathrm{a}}$ Detailed information on each mutant database can be found in Additional file 6: Table S4. 


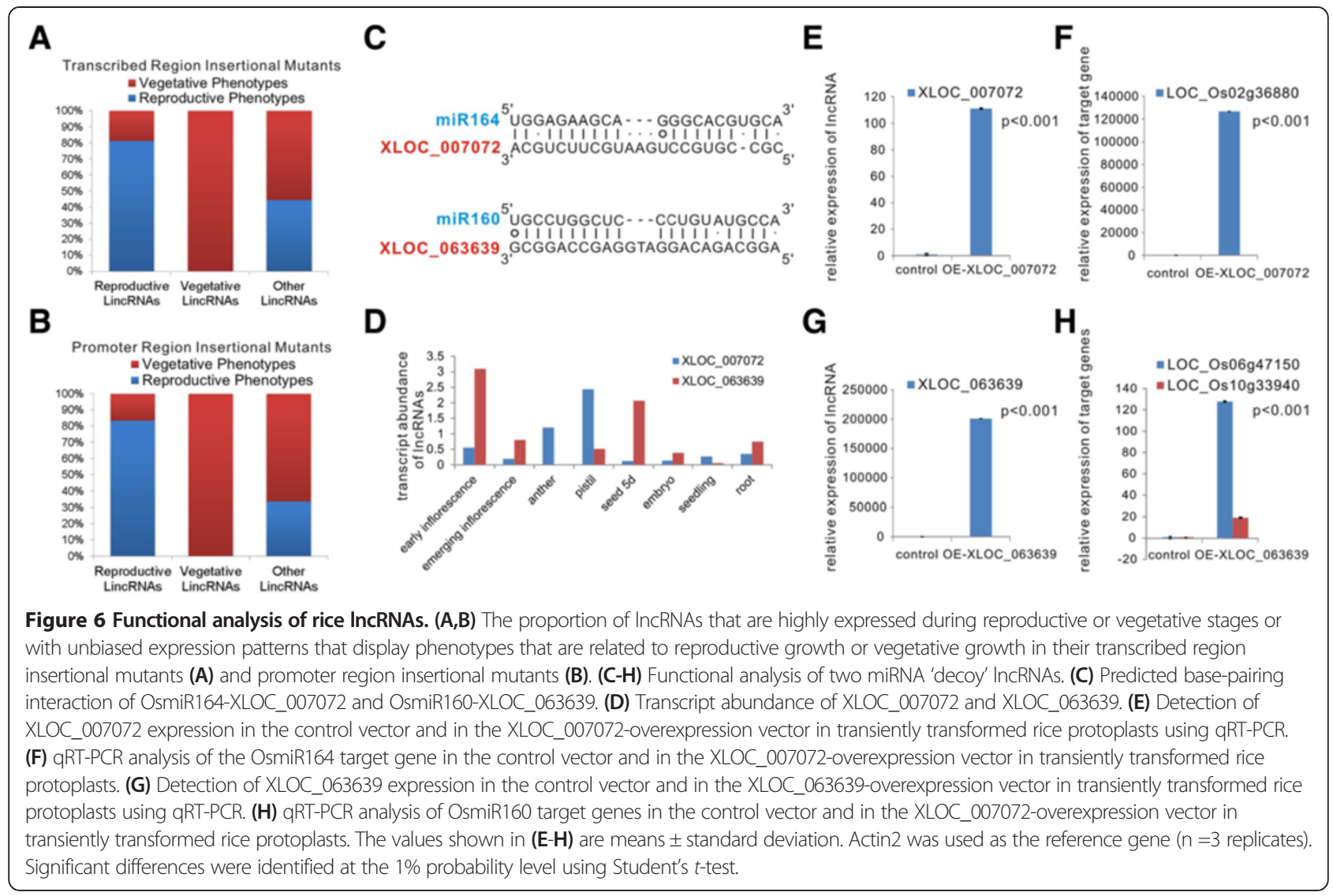

specific miRNAs in a type of target mimicry to protect the target mRNAs from repression in both plants and animals [41,42,51-57]. Because many miRNAs have been reported to regulate reproduction in plants [58], we predicted lncRNAs that might act as ceRNAs using the algorithm developed by Wu et al. [42]. Interestingly, 65 of the identified rice lincRNAs were predicted to be 'decoys' of conserved miRNAs, such as miR160, miR164, miR168, miR169 and miR408 (Additional files 9 and 10). We further used a transient transformation assay to test whether these lncRNAs could function as miRNA decoys. Expression vectors under the control of the $35 \mathrm{~S}$ promoter containing a decoy lncRNA (XLOC_0063639 or XLOC_007072) that is highly expressed during the reproductive stage were introduced into rice protoplasts separately (see the Materials and methods section for details). Twenty-four hours after transformation, the total RNA of the protoplasts was extracted, and the relative expression level of the lncRNAs and the endogenous target genes of the corresponding miRNAs were measured by qRT-PCR. Both XLOC_0063639 and XLOC_007072 dramatically increased the mRNA abundance of corresponding miRNA (OsmiR160 and OsmiR164) targets (LOC_Os02g36880 for miR164 [59]; LOC_ Os06G47150 and LOC_Os10g33940 for miR160 [60]) in their transiently expressed protoplasts, suggesting that XLOC_0063639 and XLOC_007072 indeed inhibited the functions of OsmiR160 and miR164, respectively (Figure 6C,E-H). It is known that OsmiR160 and OsmiR164 participate in regulating floral and seed development in plants [61-63]; interestingly, XLOC_007072 is specifically expressed in pistil and anther, and XLOC_0063639 is highly expressed in early panicles and seeds after pollination (Figure 6D). Thus, these two miRNA-lncRNA functional pairs might be important regulators of floral and/or seed development. Further studies are necessary to investigate the functions of these two lncRNAs in sequestering miRNAs in vivo.

We also studied a lncRNA (XLOC_057324) that is highly expressed in reproductive organs in relation to its physiological function in rice plants. First, we confirmed the expression pattern of this lncRNA using qRT-PCR and in situ hybridization. The results showed that this lncRNA is specifically expressed in young panicles and pistils (expression was restricted to ovules), suggesting that XLOC_057324 might play a role in regulating panicle and/or pistil development (Figure 7A,B). A rice mutant from the rmd database [50] that contains a T-DNA insertion in the IncRNA, XLOC_057324, was used for further functional analysis (Figure $7 \mathrm{C}$ ). We first reidentified the T-DNA insertional site and then analyzed the expression of XLOC_057324 and the phenotypes caused by the insertion. Note that no gene is located 

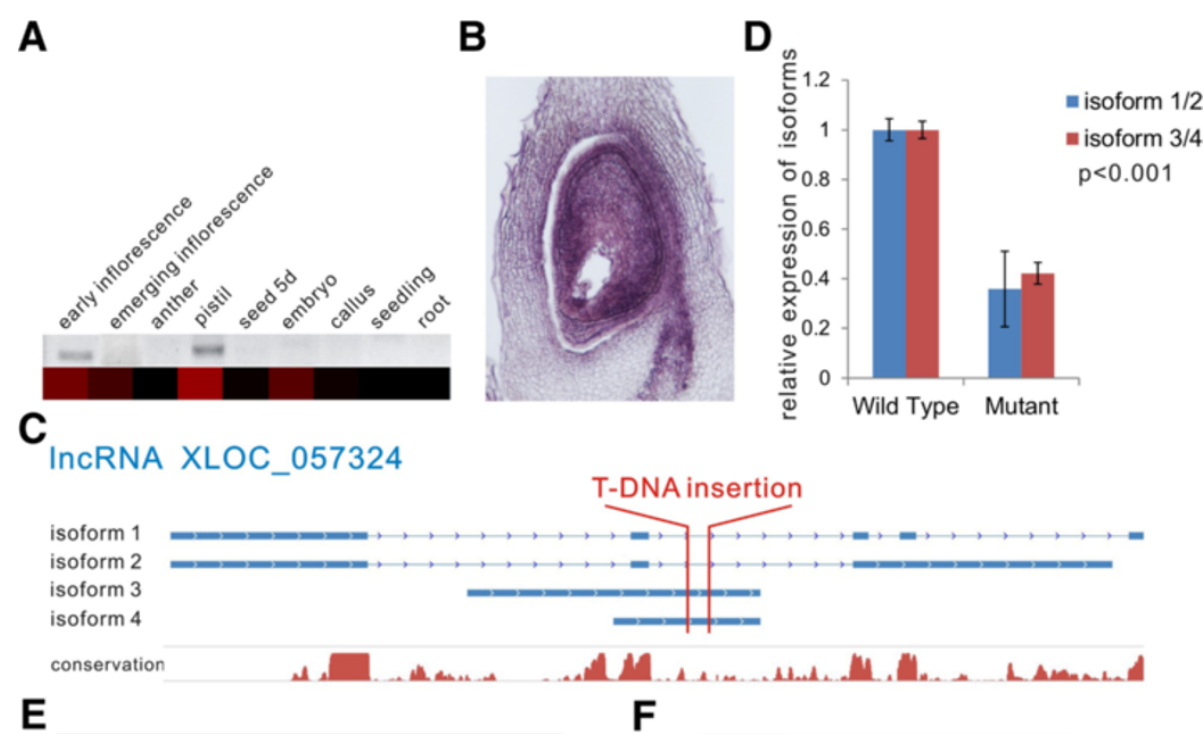

E

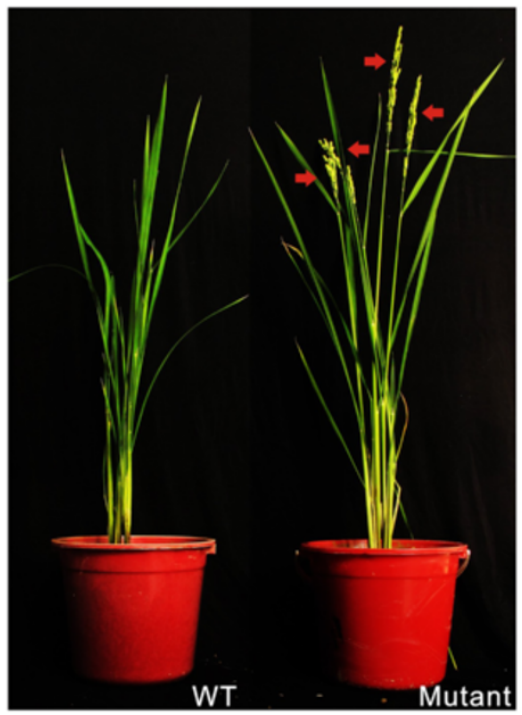

$\mathbf{F}$

G
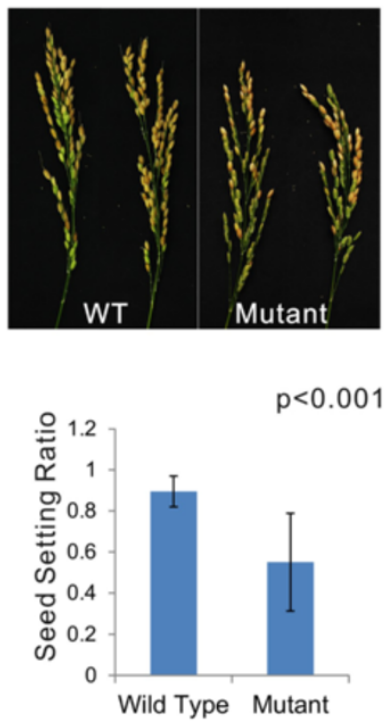

Figure 7 Functional analysis of IncRNA XLOC_057324. (A) Confirmation of the expression pattern of XLOC_057324 using semi-quantitative PCR. A heatmap was generated from the FPKM values and was used to visualize the IncRNA expression pattern in the RNA-seq data. (B) Spatial expression pattern analysis of XLOC_057324 based on in situ hybridization. (C) Isoforms and conservation of XLOC_057324; the T-DNA insertional site of the mutant analyzed in this study is marked in red. (D) Relative expression levels of XLOC_057324 isoforms in the mutant plants detected using qRT-PCR. (E) Phenotypes of wild-type (WT) plants and mutant plants during flowering; panicles are marked using red arrows. (F,G) Panicles (F) and seed setting ratios (G) of wild-type and mutant plants after harvest. The values shown in $(\mathbf{D}, \mathbf{G})$ are means \pm standard deviation; $=3$ replicates in (D); $n=20$ plants in (G). Actin2 was used as the reference gene. Significant differences were identified at the $1 \%$ probability level using Student's t-test.

$15 \mathrm{~kb}$ downstream of the T-DNA insertional site, and although one gene (LOC_Os08g35520.1) is located upstream of the insertional site, we did not detect any expression nor visible difference in the expression of this gene between wild-type plants and mutant plants (Figure S1E in Additional file 4). Thus, the mutant phenotypes are most likely to be caused by the effect of the insertion into the lncRNA XLOC_057324. This mutation apparently reduced the abundance of all isoforms of this
lncRNA (Figure 7D). As the mutant plant was generated using the japonica rice varieties Zhonghua 11 (ZH11), we further compared the phenotypes of the mutant plants with the ZH11 wild-type plants. Interestingly, the T1 and T2 mutant plants all flowered earlier than the wildtype plants when these plants were grown at the same time (Figure 7E), but the fertility decreased significantly (Figure 7F,G), indicating that XLOC_057324 is involved in panicle development and sexual reproduction. 


\section{Discussion}

Sexual reproduction is a crucial step in the life cycle of plants and is dominant among angiosperms; sexual reproduction is more crucial for crop plants because of its applications in agriculture. Over the past decade, genetic screens have identified a number of genes involved in sexual reproduction; however, the regulatory pathways that mediate the specification of reproductive organs and the process of embryogenesis are far from being understood. The recent discovery of lncRNAs has filled gaps in our knowledge of certain reproductive regulatory pathways. Although an increasing number of reports indicate that IncRNAs function in the regulation of reproduction in mammals, the identification of such lncRNAs in plants was just beginning, and only few plant lncRNAs have been shown to play roles in regulating reproductive processes [7-10]. In this study, we systematically identified and analyzed rice lncRNAs to find novel lncRNAs associated with sexual reproduction. A source of lincRNA-associated mutants was also provided to facilitate further functional analyses of rice lincRNAs. Moreover, we identified that several lncRNAs that are highly expressed in reproductive organs function as ceRNAs or participate in rice flowering and fertility processes. A number of lncRNAs were found for the first time to be specifically expressed during the reproductive stage and involved in reproduction.

IncRNAs have previously been identified in several species [13-16,18]. Human lncRNAs and Arabidopsis lncRNAs were selected for comparison in this study. Only characteristics that have been previously analyzed in human lncRNAs or in Arabidopsis lncRNAs were compared. Because Arabidopsis lncRNAs were identified using an entirely different method (tilling array) [16], some differences between Arabidopsis lncRNAs and rice lncRNAs might be due to the different identification methods used; for example, the tilling array method cannot be used to determine lncRNA introns, which might lead to an inaccurate estimation of the number of spliced Arabidopsis lncRNAs. However, after discarding subtle differences (such as the fact that plants produce more single-exon lncRNAs than humans, rice lncRNAs are longer than lncRNAs in Arabidopsis and human, short conserved elements are absent in plants, and differences exist in the exon numbers of lncRNAs between these three species), it is interesting that the overall characteristics of rice lncRNAs are similar to those of lncRNAs in Arabidopsis and human. For example, all lncRNAs in these organisms are shorter than mRNAs, can be spliced, are enriched in A/U and are nonconserved in sequence, indicating that lncRNAs may represent a type of conserved genes in eukaryotes that are undergoing rapid sequence evolution [26].

In addition, IncRNAs might have similar regulatory mechanisms in both plants and animals to some extent. It has been reported that lncRNAs could regulate various stages of gene expression either in cis or intrans [64]. Cisacting lncRNAs were first reported to control the expression of genes that are positioned in the vicinity of their transcription sites $[65,66]$. Soon afterwards, more and more trans-acting lncRNAs have also been discovered, which can regulate gene expression at independent loci [6,67-71]. In this study, we have found that rice lncRNAs are not more preferred to be coexpressed with their neighboring genes than protein coding genes. This phenomenon has also been shown in animals [13,26,72]. Our findings, together with previous reports in animals, suggest the dominant mechanism of lncRNAs might not occur in cis, especially lincRNAs function in both plants and animals.

It is generally considered that lncRNAs are highly tissuespecific in various species. This characteristic of lncRNAs might imply that they could function in maintaining tissue identity and in tissue development and differentiation. Interestingly, we found that rice lncRNAs are clearly enriched in anthers, similar to the finding that approximately one-third of human lincRNAs are specifically expressed in the testis, although their functions remain unclear [13]. This finding may hint at the importance of lncRNAs in male gametophyte genesis or in the regulation of reproductive growth. Komiya et al. [12] have previously reported that most MEL1-associated phasiRNAs are derived from lincRNAs that are specifically expressed in meiotic anthers and that contain miR2118 cleavage sites. MEL1, a rice AGO protein, has specific functions in the development of pre-meiotic germ cells and the progression of meiosis. This finding suggests that anther-specific lncRNAs might play roles in germ cell development or meiosis. In this study, we found that $58.0 \%$ of all of the identified rice lincRNAs are specifically expressed in anther; in addition, we identified many lncNATs in anthers, although the proportion is much less than that of lincRNAs. Thus, the enrichment of lincRNAs in the male reproductive organ suggests that lincRNAs have specific functions in male gametophyte genesis. We expect that more lincRNAs that are specifically expressed in male reproductive organs will be identified in other species in the future. The findings obtained in this study could therefore promote the functional analysis of rice lincRNAs.

In contrast to our understanding of small ncRNAs, little is known about the functions and regulatory mechanisms of lncRNAs. One intriguing mechanism is lncRNAmiRNA crosstalk. miRNAs have been reported as important regulators in plant and animal development, and some play essential roles in reproductive regulation $[58,73]$. This represents a new type of regulatory circuitry in which different types of RNAs can crosstalk with each other. In recent years, the functional target mimicries (or natural miRNA sponges) were initially discovered in plants [41], and subsequently in mammals, in which they were renamed to ceRNAs and were shown to be relevant in 
many process $[55,56,74,75]$, suggesting that these molecules might represent a widespread form of gene regulation. Some lncRNAs that contain miRNA-binding sites have been shown to communicate with and regulate corresponding miRNA target genes by competing specifically for shared miRNAs. In plants, after the IPS1-target mimic of miR399 was identified, Wu et al. [42] predicted endogenous mimics (eTMs) for 20 conserved miRNAs from intergenic or non-coding gene-originated regions in Arabidopsis and rice, and several Arabidopsis eTMs have been shown to be functional $[41,42,52]$. We also predicted that IncRNAs act as ceRNAs for conserved miRNAs in rice. After experimental verification, two of these reproduction-related IncRNAs were confirmed to be target mimics of miR160 and miR164, respectively. It has been reported that a decrease in miR160 causes abnormal flower morphology, reduced fertility and aberrant seeds and that miR164 plays a role in specifying particular cell types during the later stages of flower development [61-63]. Considering that the 'sponge' of miR160 is highly expressed in early panicles and seeds after pollination and that the 'sponge' of miR164 is specifically expressed in pistil and anther, it is intriguing to associate these two lncRNAs with the functions of miR160/miR164 in regulating floral and/or seed development. We believe that the importance of lncRNAs in their role as ceRNAs during plant development and reproduction regulation will emerge within a few years.

\section{Conclusions}

We identified 2,224 lncRNAs in rice, including both lincRNAs and lncNATs, with a focus on lncRNAs that are related to reproduction. The characteristics of rice IncRNAs were analyzed and compared with those of lncRNAs from other species. Further functional analysis showed that some IncRNAs function as ceRNAs and that one lncRNA functions as a regulator of panicle development and fertility. The research has provided a source of lncRNAs and associated insertional mutants in rice and has demonstrated the important functions played by lncRNAs in reproduction.

\section{Materials and methods}

Whole transcriptome library preparation and sequencing Total RNA was obtained from rice anthers before flowering, pistils before flowering, spikelets 5 DAP and shoots 14 DAG; these samples were used for sequencing. The preparation of whole transcriptome libraries and deep sequencing were performed by the Annoroad Gene Technology Corporation (Beijing, PR China). Whole transcriptome libraries were constructed using TruSeq Stranded Total RNA with Ribo-Zero Gold (Illumina, San Diego, CA, USA) according to the manufacturer's instructions. Libraries were controlled for quality and quantified using the
BioAnalyzer 2100 system and qPCR (Kapa Biosystems, Woburn, MA, USA). The resulting libraries were sequenced initially on a HiSeq 2000 instrument that generated paired-end reads of 100 nucleotides. The sequencing data have been submitted to the NCBI Sequence Read Archive (SRA accession number SRP047482).

\section{Data sources}

Oryza sativa genome assembly RGAP 7.0 was used throughout this study and was downloaded from [30]. All of the RNA-seq datasets used in this study were obtained from NCBI SRA. Detailed information on each RNA-seq dataset can be found in Additional file 1.

\section{IncRNA identification pipeline}

The rice transcriptome was assembled using the Cufflinks 2.0 package according to the instructions provided [19]. Briefly, each RNA-seq dataset was aligned to the rice genome independently using the TopHat 2.0 program [76]. The transcriptome from each dataset was then assembled independently using the Cufflinks 2.0 program. All transcriptomes were pooled and merged to generate a final transcriptome using Cuffmerge. After the final transcriptome was produced, Cuffdiff was used to estimate the abundance of all transcripts based on the final transcriptome, and a BAM file was generated from the TopHat alignment. All transcripts without strand information and all single-exon transcripts within a range of $500 \mathrm{bp}$ in the sense direction to other transcripts were discarded. Next, we discarded transcripts that overlapped with known mRNAs (including TE-related mRNAs) and transcripts with FPKM scores $<0.5$ ( 2 for single-exon transcripts) in all samples and transcripts shorter than $200 \mathrm{bp}$. Filtering of the remaining transcripts resulted in many novel, long, expressed transcripts. We first used the CPC [20] to predict transcripts with coding potential. All transcripts with $\mathrm{CPC}$ scores $>0$ were discarded. The remaining transcripts were subjected to HMMER [21] analysis to exclude transcripts that contained any known protein domains cataloged in the Pfam database [22]. The transcripts that remained were considered reliably expressed lncRNAs. The tissue-specific score (JS score) was calculated for each transcript using the csSpecificity() function in the CummeRbund R package [76].

miRNA decoy site prediction

miRNA decoy sites were predicted using the algorithm developped by Wu et al. [42].

\section{Whole-genome alignment}

A plant eight-way whole-genome alignment was performed according to the instructions of the UCSC Genome Browser Wiki [77]. Specifically, we first collected all of the necessary genome sequences, including those for Musa 
acuminatea (musa, v1.0) [31], Arabidopsis thaliana (Arabidopsis, TAIR9 assembly) [32], Brachypodium distachyon (brachypodium, v1.2) [33], Zea mays (maize, release 5b.60) [34], Populus trichocarpa (poplar, v2.2) [35], Vitis vinifera (grapevine, 12x) [36] and Sorghum bicolor (sorghum, v1.0) [37].

All eight genomes were masked for transposable elements and other simple repeats using the RepeatMasker program. As whole-genome alignment is computationally intensive, a Linux cluster was used for parallel computation. The phylogenic tree of the eight plants used for the multiple alignment was as follows: (((( Rice Brachypodium) Sorghum) Musa)) (Grapevine (Poplar Arabidopsis))). A phylogenic model was fitted based on the multiple alignment of the eight plant genomes using the phyloFit program [39] in the phastCons package [39]. The consScores of every base were calculated from the eight-way alignments based on the fitted model using the phastCons package.

\section{Insertion mutant analysis}

To analyze the potential functions of the identified lincRNAs, we aligned the lincRNA sequences and their $1 \mathrm{~kb}$ upstream regions (for lincRNAs without strand information, both the downstream and upstream regions were used) to the FSTs of all of the mutants in nine rice mutant databases (Additional file 6) downloaded from RiceGE [78], which maintains these data on its ftp server. We retained mutants with sequence similarity scores $>90 \%$ and a $5^{\prime}$ end located either in a lincRNA sequence or in the $1 \mathrm{~kb}$ upstream region of a lincRNA. The phenotypic information for the mutants from the Rice Tos17 Insertion Mutant Database [79] was obtained through a blast search of the original database using the identified FSTs, and phenotypic information linked to the blast results was collected manually.

\section{Gene ontology analysis}

In accordance with previous studies, over-represented functional themes present in the genomic background were mapped onto the GO hierarchy using the Cytoscape plugin BINGO [80].

\section{Confirmation of IncRNA expression via qRT-PCR analysis}

Total RNAs obtained from rice panicles both before and after heading, anthers before flowering, pistils before flowering, spikelets 5 DAP, embryos 25 DAP, calluses and the shoots and roots of 14-day-old seedlings were reverse transcribed using the PrimeScript ${ }^{\mathrm{Tn}} \mathrm{RT}$ reagent kit (Takara, Otsu, Shiga, Japan). Real-time PCR was performed using SYBR Premix Ex Taq ${ }^{\text {Tw }}$ (Takara) for amplification of the PCR products. Actin2 was chosen as a reference gene. Real-time PCR was conducted according to the manufacturer's instructions (Takara), and the resultant melting curves were visually inspected to ensure the specificity of product detection. Quantification of lncRNA expression was performed using the comparative $\mathrm{Ct}$ method. These assays were performed in triplicate, and the results are presented as the mean \pm standard deviations.

\section{In situ hybridization}

In situ RNA hybridization was performed as described previously, with minor modifications [81]. Briefly, plant materials were fixed in FAA fixative for $8 \mathrm{~h}$ at $4^{\circ} \mathrm{C}$, then dehydrated after vacuum infiltration using a graded ethanol series followed by a xylene series and embedded in Paraplast Plus (Sigma-Aldrich,St. Louis, MO, USA). Microtome sections $(8 \mu \mathrm{m})$ were mounted on Probe$\mathrm{On}^{\mathrm{TH}}$ Plus microscope slides (Fisher, Waltham, MA, USA), and lncRNAs were amplified, subcloned into the pEASY $^{\text {mu }}$-T3 (TransGen Biotech, Beijing, PR China) vector and used as templates to generate sense or antisense RNA probes. The probes were transcribed using T7/SP6 RNA polymerase. Digoxigenin-labeled RNA probes were prepared using the DIG RNA Labeling Kit (SP6/T7; Roche, Basel, Switzerland) according to the manufacturer's instructions. Photomicrographs were obtained using a bright-field microscope (Leica DM5000B).

\section{Rice protoplast preparation, transfection, and RNA extraction}

Protoplast isolation from rice green tissues was performed as previously described with some modifications $[82,83]$. Briefly, 14-day-old rice shoots were cut into approximately $0.5-\mathrm{mm}$ strips and were incubated in enzyme solution (0.4 M sucrose, $20 \mathrm{mM} \mathrm{KCL}, 20 \mathrm{mM}$ MES, $1 \%$ cellulase R-10 (Yakult Honsha,Tokyo, Japan), 0.4\% macerozyme R-10 (Yakult Honsha), $10 \mathrm{mM} \mathrm{CaCl} 2,0.1 \%$ bovine serum albumin, $100 \mu \mathrm{g} / \mathrm{ml} \mathrm{Amp)} \mathrm{for} 4$ to $5 \mathrm{~h}$ in the dark with gentle shaking (40 to $60 \mathrm{rpm}$ ). After digestion, the pellets were washed with W5 solution $(154 \mathrm{mM} \mathrm{NaCl}, 125 \mathrm{mM}$ $\mathrm{CaCl}_{2}, 5 \mathrm{mM} \mathrm{KCl}$ and $2 \mathrm{mM}$ MES, adjusted to $\mathrm{pH} 5.8$ with $\mathrm{KOH}$ ), and the protoplasts were collected by centrifugation at $1,500 \mathrm{~g}$ for 3 minutes. DNA (50 to $100 \mu \mathrm{g}$ ) was used to transfect every $1 \mathrm{ml}\left(2 \times 10^{6}\right.$ cells $)$ of rice protoplasts. Transfected protoplasts were incubated at $28^{\circ} \mathrm{C}$ for $24 \mathrm{~h}$ to allow RNA expression. Total RNA was then isolated from each sample using TRIzol (Invitrogen, Waltham, MA, USA) according to the manufacturer's instructions.

\section{Additional files}

Additional file 1: Table S1. RNA-seq datasets.

Additional file 2: Table S2. Characteristics of all of the IncRNAs identified in this study.

Additional file 3: Table S3. IncRNAs associated with small RNAs. Additional file 4: Figure S1. Properties of rice IncRNAs, related to Figure 2. (A) A/U content of the Arabidopsis IncRNA transcripts and 
various regions of protein-coding transcripts. (B) Distances of the lincRNAs, protein-coding genes and control regions from their closest protein-coding genes. The controls are random intergenic regions that were size and chromosome matched to the lincRNA set. (C) Lengths of the conserved segments in the exons of mRNAs and lincRNAs. (D) Relative orientations of the lincRNAs, protein-coding genes and control regions with respect to their closest protein-coding genes within 100,000 bases. The error bars indicate the standard deviation based on 1,000 cohorts of control regions, as described in (B). (E) Expression of LOC_Os08g35520.1 in wild-type plants and mutant plants. The total RNAs were extracted from the shoots 14 DAG and the young panicles before heading of the wild-type plants and the mutant plants, respectively, and then the expression of LOC_Os08g35520.1 was detected using RT-PCR. Actin2 was used as reference gene.

Additional file 5: Figure S2. Enriched GO terms for protein-coding genes whose expression is correlated with reproductive and vegetative lincRNAs. Significantly overrepresented GO terms based on GO molecular functions and biological processes were visualized in Cytoscape [84]. The size of a node is proportional to the number of targets in the $\mathrm{GO}$ category. The color of the node represents the significance of enrichment: the deeper the color, the higher the enrichment significance. Reproduction-specific terms in the plot are highlighted.

Additional file 6: Table S4. Detailed information on the rice insertional mutant databases used in this study.

Additional file 7: Table S5. IncRNAs with available rice mutant resources. Additional file 8: Table S6. IncRNA mutants with apparent phenotypes. Additional file 9: Table S7. IncRNAs with miRNA decoy sites. Additional file 10: Putative miRNA binding sites in IncRNAs.

\section{Abbreviations}

AGO: Argonaute; ceRNA: competing endogenous RNA; CPC: Coding Potential Calculator; DAG: days after germination; DAP: days after pollination;

FPKM: fragments per kilobase of transcript per million mapped reads; FST: flanking sequence tag; GO: gene ontology; JS: Jensen-Shannon; lincRNA: large intergenic non-coding RNA; IncNAT: long non-coding natural antisense transcript; IncRNA: long non-coding RNA; miRNA: microRNA; ncRNA: non-protein-coding RNA; PCR: polymerase chain reaction; qRT-PCR: quantitative RT-PCR; SRA: Sequence Read Archive; sRNA: small regulatory RNA; ssRNA-seq: strand-specific RNA sequencing; TE: transposable element; UTR: untranslated region.

\section{Competing interests}

The authors declare that they have no competing financial interests.

\section{Authors' contributions}

YCZ and JYL carried out the functional analysis, participated in the genome-wide screening and drafted the manuscript. ZYL, YY and JPZ carried out the validation experiments. QFL participated in the genome-wide screening and qRT-PCR. LHQ and WSS participated in the design of the study and analyzed the data. YQC conceived of the study, and participated in its design and coordination and helped to draft the manuscript. All authors read and approved the final manuscript.

\section{Acknowledgments}

We thank the support from the Guangdong Province Key Laboratory of Computational Science and the Guangdong Province Computational Science Innovative Research Team. This research was supported by the National Natural Science Foundation of China (number 91335104 and 31401352), the Science and Technology Transgenic project (2014ZX0800934B), funds from PhD Programs Foundation of Ministry of Education of China (20120171130003) and from the National Science and Technology Department and Guangdong Province (2009A020102001 and S2011020001232), and a grant from the Foundation of China Postdoctoral Science (number 2014 T70833).

\section{Author details}

${ }^{1}$ Key Laboratory of Gene Engineering of the Ministry of Education, State Key Laboratory for Biocontrol, School of Life Science, Sun Yat-Sen University, Guangzhou 510275, PR China. ${ }^{2}$ Guangdong Provincial Key Laboratory of Malignant Tumor Epigenetics and Gene Regulation, Research Center of
Medicine, Sun Yat-sen Memorial Hospital, Sun Yat-sen University, Guangzhou 510120, PR China.

Received: 1 August 2014 Accepted: 28 October 2014

Published online: 03 December 2014

\section{References}

1. Voinnet O: Origin, biogenesis, and activity of plant microRNAs. Cell 2009, 136:669-687.

2. Kim ED, Sung S: Long noncoding RNA: unveiling hidden layer of gene regulatory networks. Trends Plant Sci 2012, 17:16-21.

3. Kalantry S, Purushothaman S, Bowen RB, Starmer J, Magnuson T: Evidence of Xist RNA-independent initiation of mouse imprinted X-chromosome inactivation. Nature 2009, 460:647-651.

4. Bartolomei MS, Zemel S, Tilghman SM: Parental imprinting of the mouse H19 gene. Nature 1991, 351:153-155.

5. Umlauf D, Goto Y, Cao R, Cerqueira F, Wagschal A, Zhang Y, Feil R. Imprinting along the Kcnq1 domain on mouse chromosome 7 involves repressive histone methylation and recruitment of Polycomb group complexes. Nat Genet 2004, 36:1296-1300.

6. Rinn JL, Kertesz M, Wang JK, Squazzo SL, Xu X, Brugmann SA, Goodnough $L H$, Helms JA, Farnham PJ, Segal E, Chang HY: Functional demarcation of active and silent chromatin domains in human HOX loci by noncoding RNAs. Cell 2007, 129:1311-1323.

7. Heo JB, Sung S: Vernalization-mediated epigenetic silencing by a long intronic noncoding RNA. Science 2011, 331:76-79.

8. Ding J, Lu Q, Ouyang Y, Mao H, Zhang P, Yao J, Xu C, Li X, Xiao J, Zhang Q: A long noncoding RNA regulates photoperiod-sensitive male sterility, an essential component of hybrid rice. Proc Natl Acad Sci U S A 2012, 109:2654-2659.

9. Cho J, Koo DH, Nam YW, Han CT, Lim HT, Wook J, Hur BYK: Isolation and characterization of CDNA clones expressed under male sex expression conditions in a monoecious cucumber plant (Cucumis sativusL.cv. Winter Long). Euphytica 2005, 146:271-281.

10. Ma J, Yan B, Qu Y, Qin F, Yang Y, Hao X, Yu J, Zhao Q, Zhu D, Ao G: Zm401, a short-open reading-frame mRNA or noncoding RNA, is essential for tapetum and microspore development and can regulate the floret formation in maize. J Cell Biochem 2008, 105:136-146.

11. Zhang YC, Chen YQ: Long noncoding RNAs: New regulators in plant development. Biochem Biophys Res Commun 2013, 436:111-114.

12. Komiya R, Ohyanagi H, Niihama M, Watanabe T, Nakano M, Kurata N, Nonomura K: Rice germline-specific Argonaute MEL1 protein binds to phasiRNAs generated from more than 700 lincRNAs. Plant J 2014, 78:385-397.

13. Cabili MN, Trapnell C, Goff L, Koziol M, Tazon-Vega B, Regev A, Rinn JL: Integrative annotation of human large intergenic noncoding RNAs reveals global properties and specific subclasses. Genes Dev 2011, 25:1915-1927.

14. Guttman M, Amit I, Garber M, French C, Lin MF, Feldser D, Huarte M, Zuk O, Carey BW, Cassady JP, Cabili MN, Jaenisch R, Mikkelsen TS, Jacks T, Hacohen N, Bernstein BE, Kellis M, Regev A, Rinn JL, Lander ES: Chromatin signature reveals over a thousand highly conserved large non-coding RNAs in mammals. Nature 2009, 458:223-227.

15. Guttman M, Garber M, Levin JZ, Donaghey J, Robinson J, Adiconis X, Fan L, Koziol MJ, Gnirke A, Nusbaum C, Rinn JL, Lander ES, Regev A: Ab initio reconstruction of cell type-specific transcriptomes in mouse reveals the conserved multi-exonic structure of lincRNAs. Nat Biotechnol 2010, 28:503-510.

16. Liu J, Jung C, Xu J, Wang H, Deng S, Bernad L, Arenas-Huertero C, Chua NH: Genome-wide analysis uncovers regulation of long intergenic noncoding RNAs in Arabidopsis. Plant Cell 2012, 24:4333-4345.

17. Ben Amor B, Wirth S, Merchan F, Laporte P, d'Aubenton-Carafa Y, Hirsch J, Maizel A, Mallory A, Lucas A, Deragon JM, Vaucheret $H$, Thermes C, Crespi M: Novel long non-protein coding RNAs involved in Arabidopsis differentiation and stress responses. Genome Res 2009, 19:57-69.

18. Xin $M$, Wang Y, Yao Y, Song N, Hu Z, Qin D, Xie C, Peng H, Ni Z, Sun Q: Identification and characterization of wheat long non-protein coding RNAs responsive to powdery mildew infection and heat stress by using microarray analysis and SBS sequencing. BMC Plant Biol 2011, 11:61.

19. Trapnell C, Williams BA, Pertea G, Mortazavi A, Kwan G, van Baren MJ, Salzberg SL, Wold BJ, Pachter L: Transcript assembly and quantification by RNA-Seq reveals unannotated transcripts and isoform switching during cell differentiation. Nat Biotechnol 2010, 28:511-515. 
20. Kong L, Zhang Y, Ye ZQ, Liu XQ, Zhao SQ, Wei L, Gao G: CPC: assess the protein-coding potential of transcripts using sequence features and support vector machine. Nucleic Acids Res 2007, 35:W345-W349.

21. Eddy SR: A new generation of homology search tools based on probabilistic inference. Genome Inform 2009, 23:205-211.

22. Punta $M$, Coggill $P C$, Eberhardt RY, Mistry J, Tate J, Boursnell C, Pang N Forslund K, Ceric G, Clements J, Heger A, Holm L, Sonnhammer EL, Eddy SR, Bateman A, Finn RD: The Pfam protein families database. Nucleic Acids Res 2012, 40:D290-D301.

23. Wang H, Chung PJ, Liu J, Jang IC, Kean MJ, Xu J, Chua NH: Genome-wide identification of long noncoding natural antisense transcripts and their responses to light in Arabidopsis. Genome Res 2014, 24:444-453.

24. Derrien T, Johnson R, Bussotti G, Tanzer A, Djebali S, Tilgner H, Guernec G, Martin D, Merkel A, Knowles DG, Lagarde J, Veeravalli L, Ruan X, Ruan Y, Lassmann T, Carninci P, Brown JB, Lipovich L, Gonzalez JM, Thomas M, Davis CA, Shiekhattar R, Gingeras TR, Hubbard TJ, Notredame C, Harrow J, Guigó R: The GENCODE v7 catalog of human long noncoding RNAs: analysis of their gene structure, evolution, and expression. Genome Res 2012, 22:1775-1789.

25. Chen $C Y$, Shyu AB: AU-rich elements: characterization and importance in mRNA degradation. Trends Biochem Sci 1995, 20:465-470.

26. Ulitsky I, Shkumatava A, Jan CH, Sive H, Bartel DP: Conserved function of lincRNAs in vertebrate embryonic development despite rapid sequence evolution. Cell 2011, 147:1537-1550.

27. Nam JW, Bartel DP: Long noncoding RNAs in C. elegans. Genome Res 2012, 22:2529-2540

28. RepeatMasker Open-3.0. [http://www.repeatmasker.org]

29. UCSC genome browser. [http://genome.ucsc.edu/]

30. Ouyang S, Zhu W, Hamilton J, Lin H, Campbell M, Childs K, Thibaud-Nissen F, Malek RL, Lee Y, Zheng L, Orvis J, Haas B, Wortman J, Buell CR: The TIGR Rice Genome Annotation Resource: improvements and new features. Nucleic Acids Res 2007, 35:D883-D887. http://rice.plantbiology.msu.edu/.

31. D'Hont A, Denoeud F, Aury JM, Baurens FC, Carreel F, Garsmeur O, Noel B, Bocs S, Droc G, Rouard M, Da Silva C, Jabbari K, Cardi C, Poulain J, Souquet M, Labadie K, Jourda C, Lengellé J, Rodier-Goud M, Alberti A, Bernard M, Correa M, Ayyampalayam S, Mckain MR, Leebens-Mack J, Burgess D, Freeling M, Mbéguié-A-Mbéguié $D$, Chabannes M, Wicker T, et al: The banana (Musa acuminata) genome and the evolution of monocotyledonous plants. Nature 2012, 488:213-217. http://banana-genome.cirad.fr/home.

32. Lamesch P, Berardini TZ, Li D, Swarbreck D, Wilks C, Sasidharan R, Muller R, Dreher K, Alexander DL, Garcia-Hernandez M, Karthikeyan AS, Lee CH, Nelson WD, Ploetz L, Singh S, Wensel A, Huala E: The Arabidopsis Information Resource (TAIR): improved gene annotation and new tools. Nucleic Acids Res 2012, 40:D1202-D1210. http://www.arabidopsis.org/index.jsp.

33. International Brachypodium Initiative: Genome: Genome sequencing and analysis of the model grass Brachypodium distachyon. Nature 2010, 463:763-768. http://www.brachypodium.org/.

34. Schnable PS, Ware D, Fulton RS, Stein JC, Wei F, Pasternak S, Liang C, Zhang J, Fulton L, Graves TA, Minx P, Reily AD, Courtney L, Kruchowski SS, Tomlinson C, Strong C, Delehaunty K, Fronick C, Courtney B, Rock SM, Belter E, Du F, Kim K, Abbott RM, Cotton M, Levy A, Marchetto P, Ochoa K, Jackson SM, Gillam B, et al: The B73 maize genome: complexity, diversity, and dynamics. Science 2009, 326:1112-1115. http://www.maizesequence.org/index.html.

35. Tuskan GA, Difazio S, Jansson S, Bohlmann J, Grigoriev I, Hellsten U, Putnam N, Ralph S, Rombauts S, Salamov A, Schein J, Sterck L, Aerts A, Bhalerao RR, Bhalerao RP, Blaudez D, Boerjan W, Brun A, Brunner A, Busov V, Campbell M, Carlson J, Chalot M, Chapman J, Chen GL, Cooper D, Coutinho PM, Couturier J, Covert S, Cronk Q, et al: The genome of black cottonwood, Populus trichocarpa (Torr. \& Gray). Science 2006, 313:1596-1604. http://www. phytozome.net/dataUsagePolicy.php?org=Org_Ptrichocarpa_v2.2.

36. Jaillon O, Aury JM, Noel B, Policriti A, Clepet C, Casagrande A, Choisne N, Aubourg S, Vitulo N, Jubin C, Vezzi A, Legeai F, Hugueney P, Dasilva C, Horner D, Mica E, Jublot D, Poulain J, Bruyère C, Billault A, Segurens B, Gouyvenoux M, Ugarte E, Cattonaro F, Anthouard V, Vico V, Del Fabbro C, Alaux M, Di Gaspero G, Dumas V, et al: The grapevine genome sequence suggests ancestral hexaploidization in major angiosperm phyla. Nature 2007, 449:463-467. http:/www.genoscope.cns.fr/externe/GenomeBrowser/ Vitis/.

37. Paterson AH, Bowers JE, Bruggmann R, Dubchak I, Grimwood J, Gundlach $H_{\text {, }}$ Haberer G, Hellsten U, Mitros T, Poliakov A, Schmutz J, Spannagl M, Tang H, Wang X, Wicker T, Bharti AK, Chapman J, Feltus FA, Gowik U, Grigoriev IV,
Lyons E, Maher CA, Martis M, Narechania A, Otillar RP, Penning BW, Salamov AA, Wang Y, Zhang L, Carpita NC, et al: The Sorghum bicolor genome and the diversification of grasses. Nature 2009, 457:551-556. http://www.phytozome. net/dataUsagePolicy.php?org=Org_Sbicolor.

38. Blanchette M, Kent WJ, Riemer C, Elnitski L, Smit AF, Roskin KM, Baertsch R, Rosenbloom K, Clawson H, Green ED, Haussler D, Miller W: Aligning multiple genomic sequences with the threaded blockset aligner. Genome Res 2004, 14:708-715.

39. Siepel A, Bejerano G, Pedersen JS, Hinrichs AS, Hou M, Rosenbloom K, Clawson H, Spieth J, Hillier LW, Richards S, Weinstock GM, Wilson RK, Gibbs RA, Kent WJ, Miller W, Haussler D: Evolutionarily conserved elements in vertebrate, insect, worm, and yeast genomes. Genome Res 2005, 15:1034-1050.

40. Chen CL, Liang D, Zhou H, Zhuo M, Chen YQ, Qu LH: The high diversity of snoRNAs in plants: identification and comparative study of 120 snoRNA genes from Oryza sativa. Nucleic Acids Res 2003, 31:2601-2613.

41. Franco-Zorrilla JM, Valli A, Todesco M, Mateos I, Puga MI, Rubio-Somoza I, Leyva A, Weigel D, Garcia JA, Paz-Ares J: Target mimicry provides a new mechanism for regulation of microRNA activity. Nat Genet 2007, 39:1033-1037.

42. Wu HJ, Wang ZM, Wang M, Wang XJ: Widespread long noncoding RNAs as endogenous target mimics for microRNAs in plants. Plant Physiol 2013, 161:1875-1884.

43. Sharan R, Maron-Katz A, Shamir R: CLICK and EXPANDER: a system for clustering and visualizing gene expression data. Bioinformatics 2003, 19:1787-1799.

44. Miyao A, Tanaka K, Murata K, Sawaki H, Takeda S, Abe K, Shinozuka Y, Onosato K, Hirochika H: Target site specificity of the Tos 17 retrotransposon shows a preference for insertion within genes and against insertion in retrotransposon-rich regions of the genome. Plant Cell 2003, 15:1771-1780.

45. Miyao A, Iwasaki Y, Kitano H, Itoh J, Maekawa M, Murata K, Yatou O, Nagato Y, Hirochika H: A large-scale collection of phenotypic data describing an insertional mutant population to facilitate functional analysis of rice genes. Plant Mol Biol 2007, 63:625-635.

46. Sallaud C, Gay C, Larmande P, Bes M, Piffanelli P, Piegu B, Droc G, Regad F, Bourgeois E, Meynard D, Périn C, Sabau X, Ghesquière A, Glaszmann JC, Delseny M, Guiderdoni E: High throughput T-DNA insertion mutagenesis in rice: a first step towards in silico reverse genetics. Plant J 2004, 39:450-464.

47. Droc G, Ruiz M, Larmande P, Pereira A, Piffanelli P, Morel JB, Dievart A, Courtois B, Guiderdoni E, Perin C: OryGenesDB: a database for rice reverse genetics. Nucleic Acids Res 2006, 34:D736-D740.

48. van Enckevort LJ, Droc G, Piffanelli P, Greco R, Gagneur C, Weber C, Gonzalez VM, Cabot P, Fornara F, Berri S, Miro B, Lan P, Rafel M, Capell T, Puigdomènech P, Ouwerkerk PB, Meijer AH, Pe' E, Colombo L, Christou P, Guiderdoni E, Pereira A: EU-OSTID: a collection of transposon insertional mutants for functional genomics in rice. Plant Mol Biol 2005, 59:99-110.

49. Jeon JS, Lee $\mathrm{S}$, Jung $\mathrm{KH}$, Jun $\mathrm{SH}$, Jeong DH, Lee J, Kim C, Jang S, Yang $\mathrm{K}$, Nam J, An K, Han MJ, Sung RJ, Choi HS, Yu JH, Choi JH, Cho SY, Cha SS, Kim SI, An G: T-DNA insertional mutagenesis for functional genomics in rice. Plant J 2000, 22:561-570.

50. Zhang J, Li C, Wu C, Xiong L, Chen G, Zhang Q, Wang S: RMD: a rice mutant database for functional analysis of the rice genome. Nucleic Acids Res 2006, 34:D745-D748.

51. Liu C, Muchhal US, Raghothama KG: Differential expression of TPS11, a phosphate starvation-induced gene in tomato. Plant Mol Biol 1997, 33:867-874

52. Martin AC, del Pozo JC, Iglesias J, Rubio V, Solano R, de La Pena A, Leyva A, Paz-Ares J: Influence of cytokinins on the expression of phosphate starvation responsive genes in Arabidopsis. Plant J 2000, 24:559-567.

53. Poliseno L, Salmena L, Zhang J, Carver B, Haveman WJ, Pandolfi PP: A coding-independent function of gene and pseudogene mRNAs regulates tumour biology. Nature 2010, 465:1033-1038.

54. Sumazin P, Yang X, Chiu HS, Chung WJ, lyer A, Llobet-Navas D, Rajbhandari P, Bansal M, Guarnieri P, Silva J, Califano A: An extensive microRNA-mediated network of RNA-RNA interactions regulates established oncogenic pathways in glioblastoma. Cell 2011, 147:370-381.

55. Karreth FA, Tay Y, Perna D, Ala U, Tan SM, Rust AG, DeNicola G, Webster KA, Weiss D, Perez-Mancera PA, Krauthammer M, Halaban R, Provero P, Adams DJ, Tuveson DA, Pandolfi PP: In vivo identification of tumor- suppressive PTEN ceRNAs in an oncogenic BRAF-induced mouse model of melanoma. Cell 2011, 147:382-395.

56. Cesana M, Cacchiarelli D, Legnini I, Santini T, Sthandier O, Chinappi M, Tramontano A, Bozzoni I: A long noncoding RNA controls muscle 
differentiation by functioning as a competing endogenous RNA. Cell 2011, 147:358-369.

57. Wang Y, Xu Z, Jiang J, Xu C, Kang J, Xiao L, Wu M, Xiong J, Guo X, Liu H: Endogenous miRNA sponge lincRNA-RoR regulates Oct4, Nanog, and Sox2 in human embryonic stem cell self-renewal. Dev Cell 2013, 25:69-80.

58. Luo Y, Guo Z, Li L: Evolutionary conservation of microRNA regulatory programs in plant flower development. Dev Biol 2013, 380:133-144.

59. Fang $Y$, Xie $K$, Xiong $L$ : Conserved miR164-targeted NAC genes negatively regulate drought resistance in rice. J Exp Bot 2014, 65:2119-2135.

60. Li YF, Zheng Y, Addo-Quaye C, Zhang L, Saini A, Jagadeeswaran G, Axtell MJ, Zhang W, Sunkar R: Transcriptome-wide identification of microRNA targets in rice. Plant $J$ 2010, 62:742-759.

61. Liu X, Huang J, Wang Y, Khanna K, Xie Z, Owen HA, Zhao D: The role of floral organs in carpels, an Arabidopsis loss-of-function mutation in MicroRNA160a, in organogenesis and the mechanism regulating its expression. Plant J 2010, 62:416-428.

62. Baker CC, Sieber P, Wellmer F, Meyerowitz EM: The early extra petals1 mutant uncovers a role for microRNA miR164c in regulating petal number in Arabidopsis. Curr Biol 2005, 15:303-315.

63. Huang T, Lopez-Giraldez F, Townsend JP, Irish VF: RBE controls microRNA164 expression to effect floral organogenesis. Development 2012, 139:2161-2169.

64. Fatica A, Bozzoni I: Long non-coding RNAs: new players in cell differentiation and development. Nat Rev Genet 2014, 15:7-21.

65. Orom UA, Derrien T, Beringer M, Gumireddy K, Gardini A, Bussotti G, Lai F, Zytnicki M, Notredame C, Huang Q, Guigo R, Shiekhattar R: Long noncoding RNAs with enhancer-like function in human cells. Cell 2010, 143:46-58.

66. Marquardt S, Raitskin O, Wu Z, Liu F, Sun Q, Dean C: Functional consequences of splicing of the antisense transcript COOLAIR on FLC transcription. Mol Cell 2014, 54:156-165.

67. Tian $\mathrm{D}$, Sun $\mathrm{S}$, Lee JT: The long noncoding RNA, Jpx, is a molecular switch for X chromosome inactivation. Cell 2010, 143:390-403.

68. Ng SY, Johnson R, Stanton LW: Human long non-coding RNAs promote pluripotency and neuronal differentiation by association with chromatin modifiers and transcription factors. EMBO J 2012, 31:522-533.

69. Hu X, Feng Y, Zhang D, Zhao SD, Hu Z, Greshock J, Zhang Y, Yang L, Zhong X, Wang LP, Jean S, Li C, Huang Q, Katsaros D, Montone KT, Tanyi JL, Lu Y, Boyd J, Nathanson KL, Li H, Mills GB, Zhang L: A functional genomic approach identifies FAL1 as an oncogenic long noncoding RNA that associates with BMI1 and represses p21 expression in cancer. Cancer Cell 2014, 26:344-357.

70. Yuan JH, Yang F, Wang F, Ma JZ, Guo YJ, Tao QF, Liu F, Pan W, Wang TT, Zhou CC, Wang SB, Wang YZ, Yang Y, Yang N, Zhou WP, Yang GS, Sun SH: A long noncoding RNA activated by TGF-beta promotes the invasionmetastasis cascade in hepatocellular carcinoma. Cancer Cell 2014, 25:666-681.

71. Wang F, Yuan JH, Wang SB, Yang F, Yuan SX, Ye C, Yang N, Zhou WP, Li WL, Li W, Sun SH: Oncofetal long noncoding RNA PVT1 promotes proliferation and stem cell-like property of hepatocellular carcinoma cells by stabilizing NOP2. Hepatology 2014, 60:1278-1290.

72. Guttman M, Rinn JL: Modular regulatory principles of large non-coding RNAs. Nature 2012, 482:339-346.

73. Zhang YC, Yu Y, Wang CY, Li ZY, Liu Q, Xu J, Liao JY, Wang XJ, Qu LH, Chen $F$, Xin P, Yan C, Chu J, Li HQ, Chen YQ: Overexpression of microRNA OsmiR397 improves rice yield by increasing grain size and promoting panicle branching. Nat Biotechnol 2013, 31:848-852.

74. Rubio-Somoza I, Weigel D, Franco-Zorilla JM, Garcia JA, Paz-Ares J: ceRNAs: miRNA target mimic mimics. Cell 2011, 147:1431-1432.

75. Tay Y, Kats L, Salmena L, Weiss D, Tan SM, Ala U, Karreth F, Poliseno L, Provero P, Di Cunto F, Lieberman J, Rigoutsos I, Pandolfi PP: Coding-independent regulation of the tumor suppressor PTEN by competing endogenous mRNAs. Cell 2011, 147:344-357.

76. Trapnell C, Roberts A, Goff L, Pertea G, Kim D, Kelley DR, Pimentel H, Salzberg SL, Rinn JL, Pachter L: Differential gene and transcript expression analysis of RNA-seq experiments with TopHat and Cufflinks. Nat Protoc 2012, 7:562-578.

77. 77.Whole genome alignment howto. [http://genomewiki.ucsc.edu/index. php/Whole_genome_alignment_howto]

78. RiceGE: Rice Functional Genomic Express Database. [http://signal.salk.edu/ cgi-bin/RiceGE]

79. Rice Tos17 Insertion Mutant Database. [http://tos.nias.affrc.go.jp/index.html.en]
80. Maere S, Heymans K, Kuiper M: BiNGO: a Cytoscape plugin to assess overrepresentation of gene ontology categories in biological networks. Bioinformatics 2005, 21:3448-3449.

81. Kouchi H, Hata S: Isolation and characterization of novel nodulin CDNAs representing genes expressed at early stages of soybean nodule development. Mol Gen Genet 1993, 238:106-119.

82. Chen S, Tao L, Zeng L, Vega-Sanchez ME, Umemura K, Wang GL: A highly efficient transient protoplast system for analyzing defence gene expression and protein-protein interactions in rice. Mol Plant Pathol 2006, 7:417-427.

83. Zhang Y, Su J, Duan S, Ao Y, Dai J, Liu J, Wang P, Li Y, Liu B, Feng D, Wang J, Wang $\mathrm{H}$ : A highly efficient rice green tissue protoplast system for transient gene expression and studying light/chloroplast-related processes. Plant Methods 2011, 7:30

84. Shannon P, Markiel A, Ozier O, Baliga NS, Wang JT, Ramage D, Amin N, Schwikowski B, Ideker T: Cytoscape: a software environment for integrated models of biomolecular interaction networks. Genome Res 2003, 13:2498-2504.

doi:10.1186/s13059-014-0512-1

Cite this article as: Zhang et al:: Genome-wide screening and functional analysis identify a large number of long noncoding RNAs involved in the sexual reproduction of rice. Genome Biology 2014 15:512.

\section{Submit your next manuscript to BioMed Central and take full advantage of:}

- Convenient online submission

- Thorough peer review

- No space constraints or color figure charges

- Immediate publication on acceptance

- Inclusion in PubMed, CAS, Scopus and Google Scholar

- Research which is freely available for redistribution 